\title{
DE LA OCUPACIÓN A LA ACULTURACIÓN: ESCUELA CHILENA Y PUEBLO MAPUCHE EN LOS SIGLOS XIX Y XX*
}

\section{FROM OCUPATION TO CULTURIZATION: CHILEAN SCHOOL AND MAPUCHE PEOPLE ON THE XIX AND XX CENTURY}

\begin{abstract}
Omar Turra Diaz **
RESUMEN

oturra@wessexschool.cl

En tiempos en que el reconocimiento y valoración de la diversidad cultural se ha transformado en un discurso profusamente extendido. Este articulo exarnina cómo el sistema escolar nacional ha desconsiderado históricamente esta perspectiva en su relación educativa con la cultura mapuche. En el contexto de la formación del Estado-Nación, la escuela püblica fue diseñada, en el siglo XIX, precisamente, para homogeneizar la cultura, a partir de los intereses de los grupos dominantes de la sociedad. Este fue el principio que orientó la lógica educativa de la escuela chilena respecto a la cultura mapuche, en la que el objetivo central resultaba ser la asimilación de su población a la sociedad chilena. Sólo recientemente, a fines del siglo XX, el sistema curricular otorga consideración, al menos declanativamente, a la diversidad cultural en sus distintos niveles de concreción, lo que se constituye en una posibilidad educativa de mayor pertinencia y significación para los educandos de origen mapuche.
\end{abstract}

Palabras elave: diversidad cultural, homogeneidad cultural, escuela chilena, cultura mapuche.

\begin{abstract}
In times in which the recognition and value of cultural diversity has turned into a profusely widespread dialogue. This article examines how the school system has historically not considered this perspective, in its educational relationship with the indigenous culture. In the context of the formation of the State-Nation, the state school was precisely designed, in the 19th century, to homogenize the culture based on the interests of society's dominant groups. This was the principle that guided the oducational logic within Chilean schools with regard to the Mapuche culture, in which the main aim turned out to be the assimilation of its population into the Chilean society. Only recently, late 20th century, the national curriculum has granted consideration, at least as a statement, to cultural diversity in its different levels of coneretion, which cosstitutes an educational possibility of major relevancy for and significance to the Mapuche descendant's students.
\end{abstract}

Keywords: cultural diversity, cultural homogeneity, Chilean school, mapuche culture.

Un fenómeno social caracteristico de fines del siglo XX y que se proyecta en la actualidad, es el reconocimiento, a nivel mundial, de la diversidad cultural. Se trata de la constatación de un fenómeno que no es nuevo, sino que ha estado presente a lo largo de

la historia de la humanidad, pero que el discurso de la postmodernidad ha reconocido y valorizado como un rasgo constitutivo de las sociedades actuales. ${ }^{1}$

El redescubrimiento de la diversidad étnico-cultural constituye, sin duda, un importante cambio de perspectiva en los imaginarios colectivos de sociedades que durante siglos pretendieron eliminar, o al menos ignorar, todo aquello que la cultura dominante no pudiera comprender e incorporar bajo sus reglas morales y

\footnotetext{
- Esle articulo fue elaborado en el marco de la inveatigacion de tesis doctoral titulade Historia. Curriculum e Ldentidad. Enseriarza de la Historia y Ciencias Sociales en contexto educacional mapuche que el autor realizz en el Programa de Doctorado en Educación de fa Universidad de Concepción. Profesor Guia: De. Leonardo Mazzei de Grazia.

* Profesor, Magister en Historia, The Wessex School, Concepción, Chite

1. Hay autores que consideran que el discurso de la modernidad, caracterizado cumo un discurso begemónico, unificador y totalizante, propio de la razón iluminista e instrumental, estatia agotado $y$, con ello, se ingresaria a una nueva época. posterior a la modernidad. Otros, sefialan que esta nueva época seria una expresión y. por tanto extersión de la modernidad. Para mayor profundización véase, Lyotard, lean Francois, 1987. La condicion Posmoderna, Madrid, Ed, Catedra; Roa. Armander 1995. Modernidad y Postmodertidad, Santiago, Ed. Andrís Bello.
} 
sus códigos de poder. No obstante, frente a la histórica pretensión de extinguir las expresiones culturales que no se ajustaran a los cánones de la cultura oficial, surgen en diferentes lugares del planeta, movimientos nacionalistas, separatistas y corrientes intelectuales que reivindican la importancia de preservar todas esas formas de expresión de pueblos, etnias y grupos humanos a partir de las cuales se construye la riqueza cultural de la humanidad.

Si bien resulta una verdad incuestionable que, prácticamente, todas las sociedades han sido multiculturales en función de sus complejos desarrollos históricos, la diversidad cultural se ha multiplicado debido a las corrientes migratorias, intra e intercontinentales, $y$ ha adquirido relevancia a partir de las presiones sociopolíticas relacionados con los procesos democráticos y el resurgir de las demandas identitarias de las minorias étnicas en los diversos Estados. Tales fenómenos se desarrollan con especial intensidad durante la segunda mitad del siglo XX.

Si bien el discurso de la diversidad cultural se ha extendido profusamente a nivel social, pareciera ser que su incorporación al sistema educativo genera una serie de tensiones con la estructura y función social misma del sistema escolar y los currículos nacionales. Estas tensiones se relacionan con el rol que el sistema social le ha asignado históricamente a la educación y, con ello, a su estructura curricular como un instrumento que permita lograt la meta de un ciudadano que se integre productivamente a la sociedad, según los requerimientos de la cultura dominante.

\section{ESTADO NACIONAL, EDUCACIÓNY HOMOGENEIDAD CULTURAL}

Para entender este fenómeno social en nuestro espacio cultural, que se expresa en la dicotomia homogeneidad-diversidad cultural, es necesario examinar la función sociocultural que el sistema de poder, históricamente establecido, ha asignado a la educación en las sociedades hispanoamericanas. ${ }^{2}$

En este sentido, el rol fundamental que se dio a la educación en la época colonial, fue precisamente el de civilizar a quienes no compartian los códigos culturales propiciados por los colonizadores. En esta etapa, las escuelas de indios que se establecieron, en la generalidad de los territorios conquistados, cumplieron un papel fundamental en la tarea de sustituir las lenguas nativas por el español y la enseñanza del catecismo como herramienta para borrar las religiones originarias. ${ }^{3}$

El propósito civilizador de la escuela hispanoamericana pretendia alejar a las poblaciones indigenas de sus concepciones ancestrales, para prepararlos en las pautas de la cultura dominante. Entendamos que, en este contexto, civilizar significa occidentalizar, imponer los valores de los conquistadores curopeos que a partir del descubrimiento se aventuraron hacia estas latitudes.

De manera general se puede señalar que en su proyecto civilizador los europeos dispusieron de tres grandes herramientas: armas, religión y educación. Las armas definieron las relaciones de poder, el cristianismo fijó el nuevo orden de los valores y creencias, y la educación sentó las bases para la uniformidad cultural. ${ }^{4}$

En esta lógica homogeneizadora, la escuela en la época colonial desconsideró lenguas y dialectos, costumbres y vestuarios, conductas, creencias religiosas y conocimientos ancestrales, para subordinarlos a los conocimientos universales provenientes de la concepción filosófica y cientifica de Occidente, a la lengua y religión oficial de los conquistadores. La diversidad debe ser reducida a su minima expresión para instituir, por el contrario, mecanismos de simplificación, disciplina y control colonial.

El fenómeno histórico de la conformación de los Estados Nacionales que surge a partir de la Revolución Francesa, viene a reafirmar la función homogeneizadora de la escuela hispanoamericana, pues debe responder al interés de las nuevas elites gobernantes por promover saberes $y$ valores, que permitan una formación común para los integrantes de estas incipientes sociedades.

Hispanoamérica desarrolla en el siglo XIX procesos de emergencia de formas politicas autónomas orientadas a despojarse del pasado colonial español. Las elites oligárquicas criollas, que encabezaban este proceso en las distintas latitudes del antiguo imperio español, sustentan el proyecto fundacional de las futuras

\footnotetext{
a Los sistemas educativos hispanoamericanos, con diversas graduaciones y profundidad, han debatido y desarrollado algunas lineas de acción frente al tema de la diversidad cultural en las aulas. En nuestro medio el tema también es de relevancia, desde la decada de los noventa, y una muestra de ello es que la Revista de Educación de la Pontificia Universidad Catolica. Pensamiento Educativo, le dedicara un numero especial en su volumen 3 ?.

3 Cajlao, Francisca. 2005, "Educación, escuela y diversidad", en Pensamiento Edacativo, vol. 37, PUC, Santiago, pp, 27.37.

4 Ibid. p. 29.
}

Revista de Historia, año 18, vols, 18-19, 2008-2009, pp, 213-222. 
naciones en los ideales ilustrados recogidos y apropiados en los salones y academias europeas, Los nacientes Estados se presentaban como un espacio social en que se podia articular el imaginario social moderno, dada su reciente formación y su "necesidad" de integrarse al orden mundial capitalista encabezado por Europa.

En el proyecto de conformar perfiles de subjetividad que hicieran viable la lógica de la civilización y el sueño modernizador de las elites criollas, resultaba implícito la conformación de un orden identitario homogéneo que organizara la comprensión del mundo en términos de inclusiones y exclusiones, que legitimara y aprobara aquello que contribuiria a la sociedad proyectada. ${ }^{5}$ En este sentido, la consigna era "una cultura, una nación". Esta fórmula implicaba reconocer unilateralmente como válida y legítima una formación cultural, que se identificaba con la nación y la institucionalización estatal de la negación del "otro cultural" 6 .

El Estado es el encargado de desarrollar el proceso de conformación nacional, generándose asi un estrecho vinculo que une a estas dos construcciones históricas. La nación resulta una construeción histórica que conforma una unidad expresada en el "nosotros" cultural y el Estado constituye la expresión política de tal unidad.

Intelectuales, políticos y ensayistas latinoamericanos se encargan de establecer los patrones de identidad del ciudadano nacional, que se pretende conformar mediante la configuración de un imaginario dicotómico excluyente: el imaginario de la "civilización y la barbarie" 7 El referente empirico establecido por esta configuración discursiva, identificaba la civilización con las sociedades europeas donde reinaba la urbanidad, el estado de derecho, el cultivo de las ciencias y las artes. La barbarie, por su parte, se identificaba con las sociedades indigenas americanas, dadas sus formas culturales de estrecha ligazón con la naturaleza, ruralidad y superstición y, ausencia de arte, ciencia y escritura.

Al igual que en los tiempos del dominio español, los imperativos del Estado-Nación que se estaba conformando, en el siglo XIX hispanoamericano, confrontaba directamente a las culturas indigenas originarias, degradando ontológicamente sus formas culturales. A partir de la vinculación de sus prácticas culturales con la barbarie, se desarrollan y justifican procesos históricos como la asímilación cultural, la expropiación de sus tierras, la dominación política e inclusive, en algunos casos, el exterminio étnico.

En este contexto, los sistemas educativos hispanoamericanos, como casi en todo el mundo, nacieron como instrumento para la consolidación de los estados nacionales. La diversidad cultural se consideraba una amenaza para el Estado-Nación. La unidad cultural, por el contrario, permitiría consolidarlo y de ahí la importancia de la educación. La escuela representaba la puerta de entrada al manejo de una lengua común y a los valores de la cultura dominante, Su propósito fue, claramente, homogeneizar la cultura. ${ }^{8}$

La configuración de un tipo deseable de subjetividad encuentra en el sistema educativo su verdadera posibilidad de realización. Este principio socioeducativo de plena aceptación en la actualidad, fue percibido y fomentado por los proyectistas sociales decimonónicos, quienes influidos por la llustración y el positivismo insistian en que una educación pública masiva debia compensar las debilidades de la raza. Todo mejoramiento social, señalaban, dependia de la habilidad del estado para reemplazar su legado cultural por medio de la inmigración y la masificación de la educación científica. ${ }^{9}$

La escuela, desde entonces, es asumida como un espacio de construeción sociocultural en donde se forma ese tipo de sujeto, que los ideales nacionales reclamaban:

"Lo que se busca es introyectar una disciplina sobre la mente y el cuerpo que capacite a la persona para ser 'útil a la patria'. El comportamiento del niño deberá ser reglamentado y vigilado, sometido a la adquisición de conocimientos, capacidades, hábitos, valones, modelos culturales y estilos de vida que le permitan asumir un rol "productivo" en la sociedad", 10

\footnotetext{
3 Hopenhayn. Martín, 2002 "El reto de las identidades y la multicultunalidad", en Pensar lberoamerica, Revista Digitaf de Caltura, Ne 0, httpd fwww. campus-oei org/pensariberoamerica/rico0a0i htm

s La negación del "otro cultural, indigena, implica la invisibilización de la diferencia étnica y conlleval a rivel cultural fenomenós como la deslegitimación de la afirmación identitaria indigena y la sculturación.

7 En este sentido, ta obra de Domingo Faustino Sarmiento, Facundo, civilización o barbarie, escrita en Chile, a mediados del siglo XIX, representa el inicio de este paradigma dicotomice. Para un examen màs detallado de estas ideas cfr. Kaplan, Marcos. 1983. Formatción del estado nacional en

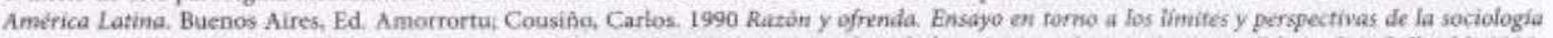
en America Latina. Santiago. PUC. Larrain. Jorge. 1996. Modemidad, nazón e identidad en América Latina. Santiaga, Ed. Andrés Belló Id. 2005. ¿América Latina moderna? Globalizacién e fdentidad. Santiago. Ed. LOM.

3. Schmelkes, Silvya. 2005. "Educar en y para la diversidad; en Pensamiento Educativo vol 37. PUC. Santiago, pp. 39-51.

7. Larnain. Jorge. 1996, p. 148.

19 Castro-Gómez, Santiago. 2003. "Ciencias sociales, violencia epistemica y el problema de la "invención del orro". en Edgardo Lander (comp.), La colonialidad del saber: eurocentrisme y ciencias sociales. Perspoctivas latinoamericanas, Buenos Aires, Ed, C1.AC.SO, p. 149.
} 
El fenómeno educativo, desde esta perspectiva, actúa como dispositivo ideológico del Estado que contribuye a la formación de ciudadanos útiles y con conciencia nacional. Especial importancia adquiere, en csta función, el curriculum escolar, como instancia educativa orientada a asegurar la transmisión de la cultura legítima y el conocimiento acumulado a las nuevas generaciones, a través de la construcción y sistematización de las diferentes áreas del saber y del quehacer humano.

El sistema educativo diseña un plan de formación en donde se transmiten valores, saberes, conocimientos y una lógica de pensamiento que se relaciona con una concepción de sociedad y cultura. ${ }^{11}$ El curriculum representa lo que se debe enseñar, lo deseable para las nuevas generaciones, es el entramado mediante el cual, la escuela intenciona las orientaciones ideológicas acerca del patrimonio cultural y sociedad deseada.

En este sentido, Magendzo, un especialista en temas curriculares, expone:

"... podemos decir que en América Latina se ha transmitido un esquema de significaciones y representaciones simbólicas y se ha comunicado, perpetuado y desarrollado oficialmente un sistema de concepciones, de conocimientos y actitudes frente a la vida que corresponden a los grupos culturalmente dominantes de la sociedad"; y agrega "El tejido discriminatorio y de negación de la diversidad cultural se forma de una manera muy sutil, a veces en la conjunción de una serie de variables históricas, sociales y psicológicas. La educación es parte importante de este tejido "./2 Históricamente, el curriculum escolar del Estado Nacional hispanoamericano consecuente con los ideales de la modernidad y los propósitos de afirmación nacional, desarrolla una politica educativa asimilacionista hacia las culturas indigenas. Esta se presenta como una forma de negación de la diversidad cultural indigena, negación de su propio universo simbólico con el fin de disciplinarlos en el trabajo productivo, la ideología del Estado-Nación, el espiritu racionalista y el manejo de la lengua castellana.

El currículum nacional homogéneo, en esta lógica eurocéntrica, viene a significar respecto de las culturas indigenas la invisibilización de la diferencia y el desarrollo de fenómenos de aculturación. 13

\section{ESCUELA CHILENA Y CULTURA MAPUCHE}

En Chile, la construcción del Estado-Nación se enmarca en la misma lógica que venimos señalando. La admiración por Europa y lo europeo marcó profundamente la conciencia de nuestras elites dirigentes Es asi, como desde la emancipación, una serie de pensadores impregnados del enciclopedismo europeo, postulan los caminos de la civilización y el progreso con los parámetros del pensamiento ilustrado como contrapartida a la tradición y al sustrato cultural del pais. ${ }^{14}$

A medida que se ahondaba cn las proyecciones nacionales y consolidaba la organización estatal, se hizo cada vez más urgente definir el componente poblacional que se queria, y actuar directamente sobre la base social que se deseaba cambiar. Surgia, entonces la necesidad de hacerse cargo del tema mapuche. En poco tiempo, se elaboró, al decir de Pinto, una verdadera "ideología de la ocupación", de fuerte contenido antiindeginista que impulsó y dio legitimidad al accionar del Estado, en base a la propagación de un discurso que enfatizaba en la idea de un mapuche de barbaridad incorregible, que ultrajaba al país y entorpecia su desarrollo. ${ }^{15}$ Surgia aqui, una situación paradojal a nivel del discurso de los constructores del Estado en Chile: por un lado eran valorados por su valentia, capacidad guerrera $y$, sobre todo, por su apego a la libertad, que servia de ejemplo y legitimación de la lucha contra el pasado español; pero al mismo tiempo se diseñaban estrategias para disponer de sus territorios, puesto que sus formas culturales resultaban la negación de los ideales de progreso y civilización aspirados. 16

11 Conceptualmente, quien con mayor profundidad aborda esta temática, desde el mundo angloamericano, es Michael W. Apple. Al respecto véase 1996. Jdeologia y curriculo, Madrid, Ed. Akal-Universitaria; 2001. Politica cultural y eilucación. Madrid, Ed. Morata.

13 Magendzo, Abraham. 1996. Curriculum, educación para la democracia en La modernidad. Bogota. Ed. PIIE - Instituto para el desarrollo de la democracia Luis Carlos Galin, p. 186.

13 Id. Peralta. Maria. 1996. Curriculos edacacionales en América Latina, su pertinencia cultural. Santiago, Ed. Andrés Bellos, Quifacueo, Daniel, Quintriqueo, Segundo, Cardenas, Prosperino 2005. Edwcaciön, cuericulum e interculturalidad, dementos sobre formación de profesores en contexto mapuche. Temuco, Ed. Universidad Catolica de Tenuco.

14 Cousino, op. cit: Subercaseaux. Bernardo 1996. Chilezun pals modernor Santiago. Ed. B

13 Pinto, Jorge. 2000. De la inclasión á lat exciusión, la formación del estado Ja nación y el pueblo mapache. Santiago, Colección IDFA, Ed, USACH, p. 30.

Ie Herrera, Francisca. 2003, "El discurso de la modernidad y el reclamo Mapuche' en Cyber Humanitas, Nn 27, Santiago http: Wwwwicyberhumanitatis. uchile. cl/CDA. La paradoja enunciada, se hizo presente de manera permanente en el debate acerca de la pertenencia o no de los mapuche a la nación chilena. Con el tiempo, se fue estableciendo consenso hacia la incorporación-exclusión definitiva. El mayor conocimiento que se va adquiriendo del territorio mapuche señala la visualizactón de sis potencialidades productivas y el surgimiento de los intereser econónicos por la explotación

Revista de Historia, año 18, vols, 18-19, 2008-2009, pp, 213-222. 
Como en los tiempos coloniales, las autoridades gubernamentales del siglo XIX consideraron que la educación era un excelente medio para transformar al mapuche de su barbarie a las bondades de la civilización, y de paso, favorecer la ocupación de su territorio.

El gobierno central adoptó como estrategia educativa, en el transcurso de la primera mitad del siglo XIX, el restablecimiento de las misiones en territorio mapuche. La labor educacional que desarrollarian las misiones traeria como "consecuencia inevitable, la insensible y pacifica destruccion de la barbarie", seguin palabras del Ministro de Culto e Instrucción Pública, hacia 1849.17

EI esquema educativo misional fue desarrollado por los franciscanos, a partir de 1837 y los capuchinos, desde $18499^{18} \mathrm{La}$ eficacia de su labor fue permanentemente discutida por los sectores dirigentes metropolitanos por considerar que la acción evangelizadora que conllevaba su educación, no era suficiente y que se hacía necesario una escuela donde se enseñara no sólo rudimentos de la fe sino, también, escritura y lectura en español y nociones de aritmética. Tal situación provocó que, a poco andar, el Estado decida intervenir de manera más directa en la educación del territorio mapuche, estableciendo la escuela pública.

El establecimiento de la educación pública en la frontera mapuche, según Serrano, se inició desde la década de 1850, al margen del tema indigena, es decir, no estaba concebida para educar especificamente a la población mapuche, como si lo pretendia la escuela misional. ${ }^{19}$ Sus objetivos fueron los comunes a las zonas rurales del pais; una escuela pensada para ordenar y disciplinar a los sectores populares, de acuerdo a un patrón homogéneo para todo el país. La escuela pública formaba parte de la estrategia de colonización, del asentamiento de población chilena en la frontera, de la formación de pueblos, que lenta e imperceptiblemente terminarian por hacer desaparecer las costumbres mapuche y los integraría a la civilización.

La escuela pública se impuso rápidamente en el territorio de colonización, desplazando a la escuela misional, generando la correspondiente protesta de los misioneros por la falta de acción evangelizadora que ésta poseía. Aunque estas escuelas misionales, luchaban por mantener su existencia en el territorio, continuaban siendo el único espacio educativo concebido especificamente para los mapuche y ellas estaban en dificultades por falta de recursos y escasez de sacerdotes.

Los misioneros de la Araucanía visualizaban dos estrategias, en concreto, para cristianizar y civilizar a los mapuche y separarlos culturalmente de su medio. Una era la implementación de la educación vocacional que consistia en formar talleres en las propias misiones que les instruyeran en trabajos técnicos que los acercaran a las labores urbanas. Idea que sólo quedó como proyecto, pues no lograron obtener financiamiento para su implementación. La otra estrategia, era la educación de la mujer mapuche, iniciada hacia 1870, que si bien, según los misioneros eran más tenaces en sus costumbres que los hombres, si se les educaba desde la infancia serian más perseverantes en la fe y en la civilización. En este plano, lograron convocar algunas mujeres mapuche que fueron instruidas en casas particulares financiadas por la misión, pero su poder de acción fue restringido. 20

A pesar de los esfuerzos y peticiones de los misioneros, las escuelas misionales, como estrategia de asimilación pacífica de los mapuche, habian sido sobrepasadas por el avance de la economía agraria y del Estado chileno. En su origen, la escuela y la misión eran parte de una estrategia mayor orientada a constituirse en un núcleo aglutinador de la formación de pueblos. Eran éstos, según las autoridades, los que en definitiva lograrian la aculturación mapuche:

"El contacto inmediato con hagares poblados, y el roce de indigenas con personas civilizadas, serán un poderoso estimulo para prepararlos a los goces y hábitos de la vida social, hábitos de que no querrán desprenderse sus hijos educados bajo ese régimen ".21

Claramente, asi concebida la escuela misional no tuvo éxito en su función, pues cualquier escucla que pretendiera educar a la población indigena tenia que asentarse fuera de los espacios urbanos para captar a esta población cuya organización social y territorial estaba fundada en clanes familiares de base rural.

Ae sus recursos. Por otro lado, la admiración por lo europeo, lleva a la importación de sus estilos y modos de vida y. con el proceso de inmigraciòn coloaizadora que dispuse de tierras indigenas para su explotacion, se impotta, derechamente, al componente humano de ese continente.

I2. Serrano, Sol. 1995-1996, "De escuelas todigenas sin pueblos a pueblos sin escuelas indigenasi la educación en la Araucania en el siglo XIX: en Historia, vol. 29, Suntiago, p. 432. Esta autori trata en profundidad el tema de la educación mapuche en la centuria decimonónica, por ello, en varias de las ideas aqui presentadas nos apoyamos en ella.

in Pinto, op. cit.. p. 178.

20 Serrano, op. cit. pp. $434-435$

in libid, p. 450.

21 Ibid p. 435. Memoria del Ministerio de fusticia, Culto e Instracción Pública. 1853, 
La escuela pública en la Araucania, en tanto, habia seguido a los pueblos fundados en el proceso de colonización, resultaba una creación a propósito de estos pueblos. Por lo tanto, en su labor educacional, desconsideró la particularidad cultural mapuche, pues su objetivo se orientaba a transmitir los saberes de la cultura universal dominante y formar, en esas tierras, ciudadanos productivos e integrados a la cultura común que el Estado promovia como legitima.

\section{LA ESCUELA CHILENA EN LA ARAUCANIA DESPUÉS DE LA OCUPACIÓN}

La segunda mitad del siglo XIX, testimonia el desarrollo de un proceso histórico que deviene en una sistemática degradación de las formas culturales del pueblo mapuche en el imaginario colectivo de la chilenidad, hasta ser considerados como verdaderos obstáculos para el proceso modernizador impulsado por el Estado. Se configura e instala un discurso que legitima la ocupación de sus tierras y su exclusión del proyecto sociocultural que se pretendia conformar. ${ }^{22}$ Esta configuración discursiva tiene su expresión histórica visible en la ocupación definitiva de la Araucania, hacia 1883 y su posterior proceso de "reducción" de la población mapuche en tierras destinadas para el efecto, 23

Esta coyuntura histórica señala un hito fundamental en la relación del Estado chileno con el pueblo mapuche, cuyas implicancias se proyectan hasta la actualidad. El Estado nacional define un problema central de interés económico y geopolítico, como es la integración territorial de la Araucania; para los mapuche se trata del fin de su autonomia y el comienzo de un proceso de pauperización y forzada aculturación.

La derrota y "pacificación" de los mapuche constituyó un verdadero etnocidio que ha sido llamado, eufemísticamente, como "reducción" de la población mapuche. 24 Efectivamente, la población se reduce, miles mueren, y su territorio y sus tierras se reducen. Su sociedad y su cultura son reducidas. Se transforma por la fuerza a la población mapuche en ciudadanos chilenos, en indigenas que pueden vivir en tierras entregadas como merced $\mathrm{e}$, intencionadamente, llamadas reducciones.

La sociedad mapuche fue forzada a transformarse en una sociedad agricola de pequeños campesinos pobres en medio de una sociedad rural fundamentalmente blanca, que rápidamente se integró a los procesos de modernización del país. Su actividad económica se centró en una agricultura de subsistencia y en la ganadería a pequeña escala. 25

La "reducción" y transformación de la sociedad mapuche resulta de una proyección estatal, debidamente planificada y que el Ministro de Instrucción Pública describe con elocuencia hacia 1883:

"El avance reciente de muestra ocupación militar sobre el territorio comprendido entre la provincias de Arauca y Valdivia, provoca la acción conjunta de todos los elementos de civilización que deben transformar al salvaje araucano en un hombre util a la sociedad. Al ferrocarril y al telégrafo que llevarán el movimiento industrial y comencial a esas estensas comarcas; a la inmignación de colonos que deberán poblarla de brazos nobustos y expertos para el trabajo, será necesario unir la iglesia, la escuela y el taller, para dominar moralmente al indigena, elevando su espiritu y desarraigando de él los malos hábitos y las preocupaciones en que ha sido creado, hasfa llegar a hacer de él un hombre civilizado". 26

La politica educacional del Estado chileno hacia fines del siglo XIX estaba centrada en consolidar la educación urbana y extenderla hacia aldeas y pequeños villorrios. Una educación orientada hacia el mundo rural, propiamente tal, estaba de plano descartada. La educación indigena, por lo tanto, no tenía especificidad propia y estaba inserta en el eje campo-ciudad. Se actuaba en base al supuesto que cl mapuche, como cualquier otro campesino, debia encontrarse con la escuela en las ciudades, pueblos y villorrios, que el Estado estaba conformando para el efecto. No podia encontrarse con ella en el campo, aunque alli viviera la mayoria de los potenciales escolares.

\footnotetext{
22 Para profundizar en las caracteristicas de este discurso anti-indigenista y colonizador, vease: Martinez, Christian. 1991. "Poltticas colonizadoras de chile en el sigho XIX: la ilusión modernizadora, Araucania $1813-1913$ " en Estudies Sociales, N" 69. Sartiago, pp. 79.91: Pinto, lorge. 1992. "Crisis cconomica y expansion territorial la ocupación de la Araucania en la segunda mitad del siglo XIX" en Estudias Sociales, Ne 72 . Santiago, pp. 85-126

17. Para un estadio profundizado de este proceso histórica vease Bengoa. Jose 2000. Historia del pueble mapuche (sigio XIX y XX). Santiago, Ed, LOM, 6* edicion; Pirto. Jorge. 2000. op.cit.

14. Savedra, Alejandro 2002 Los mapuche en la sociadad citlene actual. Santiago, Ed. LOM.

15 lbid $\mathrm{pp}$. $57-65$

in Memoria del Ministerio de lusticia. Culto e Instrucción Pública. Citado por Serrano, op cit, p. 461.
}

Revista de Histopta, aìo 18, vols. 18-19, 2008-2009, pp. 213-222. 
La instrueción pública en la Araucania estaba pensada para avanzar de la ciudad a la aldea, y de ésta al villorrio, adquiriendo las escuelas de las villas la denominación de "rurales". En verdad, la escuela de campo, propiamente tal, tendria que esperar que transcurriera buena parte del siglo $\mathrm{XX}$, para su instalación en el mundo rural de la Araucania y del pais en general.

Por su condición agraria ancestral y por las reducciones rurales, que diseñó el Estado, después de la ocupación, la sociedad mapuche no formaba pueblos que hubiesen permitido una escuela propiamente indigena, por lo tanto la posibilidad de acceder a la educación quedó reducida a un grupo minoritario de educandos mapuche que debian aprender lo mismo que se enseñaba a todos los niños y jóvenes que asistian a la escuela nacional chilena. En efecto, respecto del acceso a la escuela de educandos mapuche, esta se reducia, a fines del siglo XIX y comienzos del XX, a hijos de caciques que ante las nuevas circunstancias socioeconómicas, veían ciertas ventajas en la educación de sus hijos para su relación con la chilenidad; ${ }^{27}$ y a hijos de familias mapuche que emigraron a las ciudades obligados por la pobreza de las reducciones. No obstante, al decir de las autoridades, el flujo de niños mapuche a la escuela resultaba bastante reducido:

"En efecto, los indigenas viven repartidos en los campos, y por la gran distancia que generalmente los separa de los pueblos, es muy dificil que se presenten a matricularse, no obstante que las relaciones mercantiles que los ligan a los demás habitantes han influido en que aprecien su verdadero valor $y$ deseen, por tanto, recibirla. Por otra parte, el hecho de no vivir formando centros de población, hace que no sea justificable la fundación de escuelas rurales para ellos "28

La realidad educacional local hace que ciertas autoridades, como Intendentes, Inspectores de Colonización o Protectores de Indigenas, señalen su preocupación por los problemas especificos que conllevaba la educación de la población mapuche, cuestión que en la práctica significaba revisar la política educacional uniforme que el gobierno central había diseñado para todo el pais. Así, por ejemplo, la Intendencia de Cautín en conjunto con la Inspección de Colonización, enviaron hacia 1890, un proyecto que permitiera instalar internados indigenas en las ciudades, proyecto que asumia el particularismo propio de la población mapuche y proponia un currículo especial, orientado hacia la educación práctica, de menor duración, con profesores formados para ese propósito y con textos especiales. Sin embargo, estas intenciones educativas no se concretaron oficialmente y en los hechos, los niños y jóvenes mapuche que ingresaron al sistema educativo debían acceder primero a los pueblos y a través de ellos a la escuela pública chilena.

Los testimonios de autoridades de la época evidencian la subordinación y "violencia" cultural que debian experimentar los educandos mapuche que podian asistir a la escuela chilena establecida en la Araucania. Ello es coherente con una politica de educación nacional que avanzaba, en territorio mapuche, en conjunto con la propagación de la vida urbana. 29

En el nuevo escenario socioeducativo que enfrenta el pueblo mapuche, nuevamente la escuela misional se constituirá en la única instancia educativa dirigida hacia la especificidad mapuche propiamente tal. Aunque, como a mediados del siglo XIX, su objetivo es reforzar, junto a la escuela pública, la asimilación del pueblo mapuche a la sociedad chilena.

Después de la ocupación, el trabajo misional se reorganizó, aunque manteniendo el esquema básico anterior. Los misioneros franciscanos acompañaron el avance chileno como capellanes del ejército y se instalaron en los nuevos pueblos cumpliendo sus tareas evangelizadoras. Las reducciones facilitaron la labor misional tanto por razones de seguridad como de concentración de la población mapuche. Pero como en el periodo anterior, la precariedad de los recursos se constituía en el problema central para el desarrollo de su labor educativa, dado que el financiamiento estatal cada vez resultaba más dificultoso y reducido.

Los misioneros propusieron al gobierno la creación de escuelas-talleres, que el gobierno acogió, pero que no financió, y que en definitiva fue organizada por ellos mismos. Las fuentes señalan que, al menos en tres pueblos de la Araucania, funcionaron en la escuela misional talleres de carpinteria, albañileria, sastreria y zapateria. También ellos, reclamaron la creación de escuelas en las reducciones en que enseñaran profesores mapuche reclutados entre los ex alumnos de las escuelas misionales, proyecto que tampoco fructifico ${ }^{30}$

\footnotetext{
It Bengoa senala que muchos caciques, luego de la ocupación, tuvierua que entregar un hijo como sertal de paz, los que fueron lievados a Chillin o Concepción, para ser educados. Op. cit p. 383.

I Palabras del Intendente de Cautin, hacia 1889. Citado por Serrano. Op, eit. pe 463-464

29 Serrano, op, cit. 1.468 Esta autoni piantea que, este ideario educativo se enmarca dentro de los principios del Estado liberal, opesitor a cualquier tipo de particularismo, puesto que veid en la ampliación de una educación uniforme el camino para crear una sociedad de iguales ante la ley.

so Serrano, op. cit. p. 470
} 
La escasez de recursos representó una limitante para el crecimiento de la educación mapuche desarrollada por los misioneros y con el tiempo la escuela misional se transformó, también, en una escuela preferentemente para blancos. Su reducido impacto entre la población mapuche, al decir del prefecto franciscano, se debia a que,

"Los pobres indigenas que desean aprender: tienen que hacer largos viajes para ir a las casas misionales, desprendiéndose de sus hijos la mavor parte del año. Además, las casas misionales no pueden recibir sino un reducido numero de niños indigenas, por no tener recursos suficientes para alimentarlos y vestirlos durante el año escolar ${ }^{-31}$

Las primeras décadas del siglo XX señalan la expansión de la oferta educacional en la región mapuche, puesto que además de la escuela pública y misional, la zona habia sido colonizada tanto por chilenos como por extranjeros, fundamentalmente por franceses y alemanes. Estos inmigrantes fundaron sus propias escuelas para educar a sus hijos $\mathrm{y}$, además, llegaron nuevas órdenes religiosas que establecieron escuelas para educar a la población local. Cabe pensar que toda esta creciente red educativa generó un aumento en la cobertura educacional para el pueblo mapuche, aunque no contamos con cifras concretas que nos respalden y permitan hacer una evaluación cuantitativa de tal realidad.

Claramente, la escolarización se transformó, como veremos, en el principal medio de "chilenización" de la población mapuche que emigró a las ciudades y pueblos desde sus reducciones agricolas, y también lo fue, más tarde, para los mapuche que se quedaron en sus tierras, cuando se produjo la expansión de la educación pública rural.

\section{LA EDUCACIÓN EN CONTEXTO MAPUCHE DURANTE EL SIGLO XX}

Los hijos de los caciques de los tiempos de la ocupación fueron el contingente humano que recibió con cierta regularidad la educación chilena. Esta situación, generó en ellos fuertes tendencias integracionistas que hacian que su residencia la establecieran generalmente en los pueblos. Pero en este espacio social experimentaron, también, la discriminación que la sociedad chilena les imponia. La asociación de estos fenómenos, con mayor o menor graduación, genera el surgimiento de líderes y organizaciones étnicas en los distintos pueblos y ciudades de la Araucania que se pronuncian acerca del accionar educativo estatal en contexto mapuche. $^{32}$

Desde los primeros años del siglo $\mathrm{XX}$, surgieron organizaciones mapuche que comenzaron a ser reconocidas en el debate nacional por sus propuestas y reivindicaciones. La Sociedad Caupolicán Defensora de la Araucanía fue la primera de este tipo, fundándose en 1910 y siendo presidida por Manuel Neculmán. primer profesor normalista de Temuco ${ }^{33}$ Esta entidad, activa hasta mediados del siglo XX, fue seguida por otras como la Federación Araucana, la Unión Araucana, el Frente Único Araucano, la Corporación Araucana y otras que movidas por un pensamiento desarrollista sefialaban que la pobreza era la principal vestimenta de las desgracias mapuche. ${ }^{34}$

Las organizaciones mapuches, aún cuando expresaron diferencias en sus propuestas, se inclinaron por favorecer la educación. Para ello, desde los albores del siglo XX, demandaron al Estado la construcción de escuelas y cupos para estudiantes mapuches en escuelas normales y otros establecimientos, y que estos se establecieran tanto en los pueblos, como en las comunidades. Un rol central en la promoción de la educación cumplieron los profesores mapuches. Al respecto, Foerster y Montecino señalan:

Desde Manuel Neculmán, primer dirigente y fundador de la sociedad Caupolicán, una serie de maestros indigenas fueron adalides de la causa de su etnía. Estudiaron en las escuelas normales y formaron parte de los que podriamos denominar un estrato intelectual mapuche. Por eso no fue extraño que los grupos organizados incluyeran en sus demandas, -desde muy temprano- el derecho a la educación y que vieran en ella un elemento fundamental para el desarrollo de su pueblo. 35

\footnotetext{
31 Memoria del Ministerio de RR. FE. Sección Culto, 1897. Serrano, op cit. p. 470.

12 Bengoa, Op, cit, p, 383.

33 Drid, p.371.

4 Canales, Pedro, 1998, "Escuelas chilenas en contextos mapuche, integracion y resistencia, 1868-1950"en Otrima Decada, N" 9, CIDPA, Valparaiso, pp. 1-10.

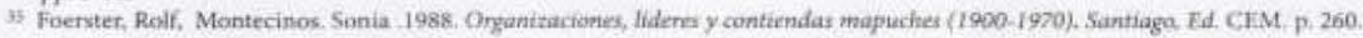

Revista de Historia, año 18, vols. 18-19, 2008-2009, pp. 213-222. 
Este planteamiento ante la educación corresponde, como señalan los autores, a un estrato intelectual mapuche que se insertó en el sistema educativo formal y abogó por el acceso a la educación escolarizada de las nuevas generaciones de jóvenes de la etnia. Los profesores mapuche fueron generando un discurso en torno a las contradicciones y diferencias respecto del mundo no-mapuche, e insistian en la idea de acercar la escuela a las comunidades y la necesidad de preparar profesores de la etnia. De la escuela se esperaba que proporcionase a los educandos mapuche:

.los medios materiales que hagan verdadenamente una labor social con los padres de familia y vecinos, es decir, dentro de las neducciones en que se halle situada y, si fuera posible, fundar colegios internados en el campo mismo, que estén en contacto con sus tierras, de actuerdo al ambiente y sus intereses y cuyos maestros fueran de su misma raza.. (y si no los fueren) que tengan vocación, cierto cariño a la raza araucana y que no sientan náuseas, repulsión considerando a los niños mapuches, al araucano nativo como un ser infeliz"36

La variedad de significados que la educación asumia para la sociedad mapuche y las consecuencias de su implementación, en este contexto socio-cultural, ha sido materia de controversia para sus integrantes. Según Marimán, el tema se ha debatido, desde principios del siglo XX, presentándose visiones contrapuestas:

"...la actitud adoptada ante su imposición fluctuó entre el rechazo y la indiferencia por parte de quienes permanecieron aferrados a su cultura, hasta aquellos que vieron en ella una herramienta viable para organizar la defensa del grupo étnico".37

El sistema educativo formal tiene para los propios indigenas un doble significado: en cuanto instrumento externo de dominación y asimilación cultural y como mecanismo de inserción cultural, laboral y social. Este significado contradictorio del fenómeno educativo entre los indigenas, provocaba rechazo $\mathrm{e}$ indiferencia en unos o posibilidades de integración y resistencia, en otros. En todo caso, la educación resulta, sobre todo en los primeros años del siglo XX, una necesidad que llevaba aparejada un alto costo cultural para la población mapuche.

Estas perspectivas se constatan en el estudio de un investigador norteamericano, Hilger, quien escribió por los años 1950, que los padres mapuche de las comunidades que estudió tenían visiones opuestas sobre la educación. Si bien, generalmente, estaban interesados en la asistencia a la escuela de sus hijos, pues reconocian la necesidad de educación formal para tratar con los chilenos, habia familias para quienes la educación formal no se consideraba necesaria, situación que era común, en muchas familias, cuando se trataba de las hijas. 38

La relación entre el Estado chileno y la sociedad mapuche se ha visualizado, en general, en función de los procesos de dominación/subordinación, que evidentemente resulta un fenómeno socio-histórico plenamente constatable, pero esta sola mirada, sugiere una pasividad de la sociedad mapuche ante la imposición de la politica educativa nacional. Las posiciones encontradas, las propuestas de los profesores mapuche, el rechazo e indiferencia de algunos, develan, también, procesos de resistencia a la asimilación y de incorporación de formas culturales de la sociedad dominante para preservar espacios de reproducción cultural propios. En este sentido, se ha planteado que los intentos de asimilación no siempre han sido exitosos, por el contrario y aunque parezca contradictorio, a pesar de la violencia de la imposición, los pueblos indigenas han sido capaces de incorporar aspectos de la educación escolar a la matriz de reproducción y producción étnica, convirtiéndola en un recurso apropiado que es utilizado y reelaborado en beneficio propio. 39

Pero en definitiva, durante el siglo XX el sistema educacional chileno en su relación con el pueblo mapuche da cuenta de sus esfuerzos de integración cultural, primero, y luego, bajo el impulso de proyectos desarrollistas, se presentó como el instrumento de cambio cultural necesario para la modificación de la estructura social y productiva de las comunidades indigenas. Ninguna de estas visiones consideró el reconocimiento a la diversidad cultural, continuando desde las políticas estatales la homogeneización de los educandos y la desconsideración de sus saberes culturales en el curriculum escolar. La escuela publica no

4. Propuesta emanada de la Concentración de Profesores de escuelas de caracter indigena de Temuca, en el año 1935 . Citado por Canates, Op, cat., p. 6.

17 Marimán, E 1997. "Demanda por educación en el movimiento mapuche en Chile 1910-1995" en Bello, A. Wilson. A. y otros Pueblas irdigenas educación y desarrollo. Temuce. Ed. Instituto de Estudios Indigenas, UFRO, p. 149

38 Citado por Pobrete, Maria Pta .2001. "Comunidades mapuches de Panguipulli y educación las primeras décadas del siglo XX" en Revista Austral de Ciencias Sociales, UACH, Ne 5. Valdivia. pp. 15-27.

39 Bello, A. y otros, Op. cit; Poblete, Op. cit. 
incorporó, entonces, el contexto cultural mapuche a la educación. La politica educacional siguió teniendo como objetivo la asimilación de los mapuche a la sociedad chilena, 40

Recién a partir de los años noventa del siglo XX el discurso educativo del Estado, respecto de las culturas indigenas, y en particular de la mapuche, señalan el reconocimiento y valoración de sus especificidades culturales, puesto que estos grupos étricos constituyen "parte esencial de las naices de la Nación Chilena".41 Diversos sectores politicos, intelectuales y organizaciones no gubernamentales recogen y consideran las demandas de las organizaciones indigenas de una educación de mejor calidad y más contextualizada respecto a su realidad sociocultural. Esto determina que en 1993 se dicte la Ley Indigena $\mathrm{N}^{\circ} 19.253$, instrumento legal que introduce la Educación Intercultural Bilingüe, como una modalidad educativa que abre espacios de descentralización y autonomia curricular para que las minorias indígenas puedan estudiar, y con ello proyectar, sus formas culturales en la escuela. Tambièn, en este sentido, la reforma curricular iniciada en 1996 estableció en sus principios generales, el espiritu de lo dispuesto en la ley indigena, al otorgar a las unidades educativas el espacio curricular, para que consideren las realidades culturales locales en el aula.

Considerando el reconocimiento y valoración actual de la diversidad cultural, a nivel de discurso y a nivel juridico, en el sistema educativo, cabe esperar un nuevo trato educacional en contexto mapuche. El curriculum escolar actual otorga espacio a la diversidad cultural en el proceso de enseñanza-aprendizaje, estableciendo niveles de autonomia a los centros educativos para que consideren la realidad cultural local en su proceso educativo, favoreciendo con ello la pertinencia de los aprendizajes y la partícipación de la comunidad local en la definición de las metas educativas; y lo más importante, a nivel curricular, la consideración de los saberes y conocimientos indigenas en el proceso educativo.

La escuela pública, en este sentido, está llamada a inaugurar y participar de un nuevo relato en la relación educativa del Estado con el pueblo mapuche. Relato que, a nuestro entender, viene a reconocer una postura epistemológica legitima como es la consideración de la diversidad cultural en el aula y que el sistema educativo entiende como una oportunidad que puede y debe enriquecer el proceso enseñanza-aprendizaje y, conjuntamente con ello, las relaciones sociales con el culturalmente diferente.

4. Serrano, Op. cit. p. 473

4) Ley Indigena Nv 19253, Principios generales, Distio Oficial de la Republica, Santiago, Chile, 5 de Octubre de 1993.

Revista de Historia, anio 18, vols. 18-19, 2008-2009, pp. 213-222. 